REVISTA MATEMATICA de la

Universidad Complutense de Madrid.

Volumen 2. numero suplementario, 1989

http://dx.doi.org/10.5209/rev REMA.1989.v2.18104

\title{
Paracommutators - brief introduction, open problems
}

\author{
JAAK PEETRE
}

\begin{abstract}
We review the basic facts about the theory of paracommutators in $\mathbb{R}^{n}$ (sec $\mathrm{S}$, Janson, J. Peetre, Trans. Am. Math. Soc. 305 (1988), 467-504). We also give an interpretation of paracommutators from the point of view of group representations. This suggests a generalization to more general groups. Here we sketch a theory of paracommutators over stratified groups. This includes the famous Heisenberg group. Finally, we take up the question of generalizing the notion of Schatten-von Neumann trace jdeals to the case of multilinear forms in (abstract) Hilbert space.
\end{abstract}

\section{INTRODUCTION}

Let us consider linear operators $T=T_{+}=T_{b}(A)$ in the Hilbert space $L^{2}\left(\mathbb{R}^{n}\right)$ given by the formula

(1) $\left.T \hat{f}\left(\xi_{1}\right)=(2 \pi)^{-n} \int_{\mathbb{R}^{n}} A\left(\xi_{1}, \xi_{2}\right) \hat{b}\left(\xi_{1}-\xi_{2}\right) \hat{f} \xi_{2}\right) d \xi_{2}$

where "stands for the Fourier transform. Here $A$ is a function, usually fixed throughout the discussion, known as the Fourier kernel of the operator and $b$ another function known as its symbol. Such operators are called paracommutators in [JP] but have also been studied by other authors, e.g. Timotin [T1], [T2], [T3], even in the case of local fields. (For some generalizations see also [QP].) As examples of paracommutators we mention iterated commutators of the type $\left[K, M_{b}\right],\left[K^{*},\left[K, M_{h}\right]\right], \ldots$, where $K, K^{*}, \ldots$ are Calderón-Zygmund operators (principal value convolutions with homogeneous functions of degree $-n$ ) and $M_{b}$ stands for the operation of multiplication with $b$. This also formally comprises Hankel and Toeplitz operators. Other examples can be found in [JP]. An extreme case is when $A \equiv 1$; then we have simply a multiplication operator. In the general case, it is helpful to think of $A$ as a kind of Schur multiplier on the Fourier side.

1980 Matematics Subject Classification (1985 revision): 47B35, 46E35, 36M35, 47D25 Editorial de la Universidad Complutense. Madrid, 1989. 
In Section 1 of this paper we briefly review the theory developed in [JPR], with complements later given by Peng Lizhong [Pen 1,2,3]. In Section 2 paracommutators are viewed upon from the formal point of view of group representations. In particular, this suggests extensions of the theory to other groups than $\mathbb{R}^{n}$, also non-Abelian ones, and, thus, in Section 3 we give a brief outline of a theory of paracommutators over stratified groups, that is, simply connected nilpotent Lie groups endowed with an auxiliary structure known as stratification. A typical example of such a group is the famous Heisenberg group. Finally, in Section 4 we mention an open question already touched upon in [Pee], [JPR]. Namely, paracommutators may also be viewed as bilinear forms and so it is natural to look at multilinear analogues too. This again calls for a theory of Schatten-von Neumann trace ideal classes for multilinear forms in (abstract) Hilbert space, which up to our knowledge is not yet fully developed.

My thanks are due to José Dorronsoro for precious information about function theory on nilpotent groups.

\section{FUNCTIONAL CHARACTER}

The theory of paracommutators is basically about finding necessary and sufficient conditions on the symbol $b$ for the operator $T_{b}=T_{b}(A)$ (when $A$ is fixed satisfying certain assumptions) to be bounded or compact or, more generally, in a suitable Schatten-von Neumann class $S_{p}, 0<p<\infty$. Recall that a compact operator $T$ in a Hilbert space is in $S_{p}$ if the sequence of its Schmidt or approximation numbers $s_{n}(T), n \geqq 0$, is in the sequence space $l_{p}$. The condition on $b$ again are expressed in terms of the scale of Besov spaces $\mathscr{B}_{p} \equiv B_{p}^{n / p, p}\left(\mathbb{R}^{n}\right)$ or related spaces such as $\mathrm{BMO}\left(\mathbb{R}^{n}\right)$. Recall that $B_{p}^{s, q}\left(\mathbb{R}^{n}\right)$, where $s \in \mathbb{R}, \hat{0}<p, q \leqslant \infty$, is a space of functions (or, if $s<0$, tempered distribution) such that, roughly speaking, one has control of $s$ derivatives in the $L_{p}$-metric, $q$ being an auxiliary parameter connected with an interpolation process. The main result in [JPR], with complements by Peng [Pen 1,2,3], has the following general character.

"Theorem". In suitable assumptions on the Fourier kernel $A$ holds $T_{b} \in S_{p}$

$n / \gamma<p \leqq \infty \Leftrightarrow b \in b_{p}$, where $\gamma>0$ is a certain number depending on $A$.

These assumptions are rather involved and in [JP] there is an elaborate system of conditions $\mathbf{A} 0, \mathrm{~A} 1, \ldots, \mathrm{A} 8$ invented to meet with various more special situations. Peng has even more conditions "interpolating" between $A n$ and $A(n+1)$. Let us however try to explain in informal terms what it is about. 
On the formal level the main assumption on $A$ is that it should be homogeneous of degree 0 or close to homogeneous. In addition it is assumed that $A$ vanishes sufficiently fast (informally, up to order $\gamma$ ) on the diagonal $\left\{\left(\xi_{2}, \xi_{2}\right): \xi_{I}=\xi_{2}\right\}$ of $\mathbb{R}^{n} \times \mathbb{R}^{n}$. This is what is needed for the "direct" part of the theorem. For the "converse" we also need some kind of nondegeneracy condition (a "Tauberian" or an "ellipticity" condition).

The proof, at least for $p>1$, follows the general pattern laid in Peller's now classic paper [Pel]; the case $p<1$ [Pen, 3] again is based on ideas of Rochberg's [R] and requires decomposition theorems for the spaces. $f_{p}$. Let us review some of the essential steps.

We begin with the easiest case $\mathrm{p}=2$. The Hilbert-Schmidt $\left(S_{2^{-}}\right)$norm of $T_{b}$ is given by

$$
\underline{\left\|T_{b}\right\| \|_{H S}^{2}}=\frac{1}{(2 \pi)^{n}} \int_{\mathbb{R}^{n}} \Lambda(\eta)|\hat{b}(\eta)|^{2} d \eta
$$

where

$$
\Lambda(\eta)=\frac{1}{(2 \pi)^{n}} \int_{\left\{\left(\xi_{1}, \xi_{2}: \xi_{1}-\xi_{2}=\eta \mid\right.\right.}\left|A\left(\xi_{1}, \xi_{2}\right)\right|^{2} d(\text { area }) .
$$

If $A$ is homogeneous of degree 0 it is clear that $\Lambda$ is homogeneous of degree $n$ but it may be infinite or zero along certain rays. As

$$
\|b\|_{-N_{2}}^{2}=\frac{1}{(2 \pi)^{n}} \int_{\mathbb{R}^{n}} \mid \eta^{\left.|m| \hat{b}(\eta)\right|^{2} d \eta} .
$$

it follows that in the optimal case, when $c_{1}\left|\eta^{n} \leqq \Lambda(n) \leqq c_{2}\right|^{n}$ with $0<c_{1} \leqq c_{2}<\infty$, the two norms $\left\|T_{b}\right\|_{H . S}$ and $\|b\|_{P_{2}}$ are equivalent.

The case $p=1$ follows from the well-known minimality of the space $\mu_{1}-$ it is the least translation and dilation invariant Banach space of functions. It is essentially a question of verifying that $T_{b}$ is in $S_{1}$ for at least one (nontrivial) symbol $b$. (This requires $\gamma<1$; to reduce to these case one has to introduce a two parameter family of operators $T^{s t}=T_{b}^{s t}=T_{b}^{y}(A)$ defined in a similar way as $T=T_{b}=T_{b}(A)$ by replacing in the definition $A\left(\xi_{1}, \xi_{2}\right)$ by the function $A\left(\xi_{1}, \xi_{2}\right)\left(1+\left|\xi_{1}\right|^{2}\right)\left(1+\left.\xi_{2}\right|^{2}\right)^{r}$.) If we then have a result about boundedness (formally $p=\infty$ ) we can by interpolation cover the direct statement for the whole range $p \in(1, \infty)$. The converse again follows by a standard duality reasoning. It makes use of the fact that the inner product in the Hilbert space.$_{2}$ provides us with a translation and dilation invariant pairing. 
Remark. It is also of interest to consider vector or operator versions of paracommutators. For instance, "big" Hankel operators in the sense of Axler [A] can in principle at least be reduced to suitable vectorial paracommutators. But, as far as we know, no general theory has yet been worked out in this case.

\section{FORMAL PROPERTIES}

We will now try to explain the deeper meaning of the assumption that the Fourier kernel $A$ should be homogeneous of degree 0 and also the very definition of the paracommutator (formula (1) of the Introduction).

Let $\zeta$ be the "affine" group, the Lie group generated by translations $\left(x \rightarrow x+h, h \in \mathbb{R}^{n}\right)$ and dilations $(x \rightarrow \delta x, \delta>0)$; it may be viewed as an extension of $\mathbb{R}^{n}$, identified with the group of translations. It acts on the Hilbert space $L^{2}\left(\mathbb{R}^{n}\right)$ via unitary transformations as follows:

$$
\begin{aligned}
& f(x) \rightarrow f(x+h), \\
& f(x) \rightarrow \delta^{n / 2} f(\delta x) .
\end{aligned}
$$

Let this representation be denoted by $U$. Then an easy calculation reveals that the family of operators $T_{b}=T_{b}(A)$ (for $A$ fixed) satisfies the formal relation

$$
U_{g} T_{b} U_{g}^{-1}=T_{\text {bog }}(g \in \zeta)
$$

Conversely, it is easy to see that any linear map $b \rightarrow T_{b}$ which satisfies (1) must be of the form $T_{b}=T_{b}(A)$ (for some $A$ ). Indeed, the distribution kernel of $T_{b}$ must be of the form

(2) $\int_{\mathbb{R}^{n}} K\left(x_{1}-y_{1} x_{2}-y\right) b(y) d y$

where $K$ is a function (or distribution) of degree $-2 n$. Taking Fourier transforms we see that

$$
A\left(\xi_{1}, \xi_{2}\right)=\hat{K}\left(\xi_{1},-\xi_{2}\right) .
$$

(For later use we remark that in terms of $K$ the Hilbert-Schmidt norm of $T_{b}$ is given by

$$
\text { (3) }\left\|T_{b}\right\|_{H . S .}^{2}=\iint_{\mathbb{R}^{n} \times \mathbb{K}^{n}} L(y-z) b(y) \overline{b(z)} d y d z
$$


where

(4) $L(y-z)=\iint_{\mathbb{R}^{n} \times \mathbb{R}^{n}} K\left(x_{1}-y, x_{2}-y\right) \overline{K\left(x_{1}-z, x_{2}-z\right)} d x_{1} d x_{2}$ )

In this way we see that paracommutators connect with group representations. In particular we can obtain the decomposition of the "regular" representation of ' $f$ s on the space of Hilbert-Schmidt operators on $L^{2}\left(\mathbb{R}^{n}\right)$ into irreducible subspaces.

For simplicity consider first the case $n=1$. Let $T$ be any Hilbert-Schmidt operator on $L^{2}(\mathbb{R})$ with (Fourier) kernel $A\left(\xi_{1}, \xi_{2}\right)$ so that the Hilbert-Schmidt $\left(S_{2}-\right)$ norm is

$$
\|T\|_{H S}^{2}=\iint_{\mathbb{R} \times \mathbb{R}}\left\langle A\left(\xi_{1}, \xi_{2}\right)^{2} d \xi_{1} d \xi_{2}\right.
$$

Introduce new variables $\eta, \tau$ with $\eta=\xi_{1}-\xi_{2}, \xi_{2}=\tau \eta$ implying $d \xi_{1} d \xi_{2}=\eta d \tau d \eta$. Then the metric is given by

$$
\|T\|_{H . S}^{2}=\iint_{\mathbb{R} \times \mathbb{R}}\left|A(\eta(1+\tau), \eta)^{2}\right| \eta \mid d \tau d \eta .
$$

Let $\left\{a_{s}\right\}$ be a fixed orthonormal basis in the Hilbert space $L^{2}(\mathbb{R}, d \tau)$. Then $A$ clearly comes as a sum

$$
A\left(\xi_{1}, \xi_{2}\right)=\Sigma A_{1}\left(\xi_{1}, \xi_{2}\right) \hat{b}_{1}\left(\xi_{1}-\xi_{2}\right)
$$

with

$$
A_{i}\left(\xi_{1}, \xi_{2}\right)=a_{i}\left(\frac{\xi_{2}}{\xi_{1}-\xi_{2}}\right)
$$

That is, $T$ comes as a sum of paracommutators

$$
T=\sum T_{b_{t}}\left(A_{1}\right)
$$

and

$$
\|T\|_{H . S}^{2}=\sum\|b\|_{B_{z}^{2}(\mathbf{R})}^{2}
$$

This is not quite the desired decomposition, but almost, as $B_{2}^{\text {s.2 }}$ comes as the (direct) sum of two irreducible subspaces: 


$$
B_{2}^{y_{2}, 2}(\mathbb{R})=\begin{array}{cl}
B_{2}^{y_{2}, 2} A(\mathbb{R}) & +\quad \overline{B_{2,2}^{y_{2}, A} A(\mathbb{R})} \\
\text { analytic functions } & \text { anti-analytic functions }
\end{array}
$$

Is it possible to choose the basis $\left\{a_{i}\right\}$ in some canonical way?

If $n>l$ we proceed similarly, the only difference being that now $e \mathscr{R}_{2}=B_{2}^{n / 2,2}\left(\mathbb{R}^{n}\right)$ comes as a continuous sum of irreducible subspaces.

\section{HEISENBERG PARACOMMUTATORS (work in progress)}

The considerations of the previous Section suggest that the theory of paracommutators can be generalized to the setting of more general groups than $\mathbb{R}^{n}$ (even non-commutative ones). What we have in mind is in the first instance stratified groups, in which case there is a natural notion of "dilation".

Recall (for more details and examples see Folland $[F]$ ) that a stratified group is a simply connected nilpotent Lie group $G$ equipped with a stratification, that is, its Lie algebra $\mathrm{g}$ is written as direct sum $\mathrm{g}=V_{1}+\ldots+V_{m}$ where the $V_{j}$ are vector spaces such that $\left[V_{i}, V_{j}\right] \subset V_{i+r}$ (That $G$ must be nilpotent follows from the existence of a stratification. Also via the exponential map $G$ and $g$ can be identified as manifolds.) The dilations $\gamma_{\delta}(\delta>0)$ are then defined (on the Lie algebra level) by the formula

$$
\gamma_{\delta}\left(x_{1} \dot{+} x_{2} \dot{+} \ldots \dot{+} x_{m}\right)=\delta x_{1} \dot{+} \delta^{2} x_{2} \dot{+} \ldots \dot{+} \delta^{m} x_{m}\left(x_{j} \in V_{j}\right) .
$$

The trace of the corresponding infinitesimal generator is called the homogeneous dimension of $G$ and will be written $Q$, i.e.

$$
Q=\operatorname{dim} V+2 \operatorname{dim} V_{2}+\ldots+m \operatorname{dim} V_{m}
$$

In [F] a theory of function spaces is worked out for stratified groups (Sobolev and Lipschitz spaces).

Example. The most interesting instance of a stratified group is the Heisenberg group. In this case $\mathrm{g}=\mathbb{R}^{2 n}+\mathbb{R}$, so $Q=2 n+2$, and the Lie algebra structure is given by

$$
\left[(z, t),\left(z^{\prime}, t^{\prime}\right)\right]=\left(0, \sigma\left(z, z^{\prime}\right)\right)\left(z, z^{*} \in \mathbb{R}^{2 n}, t, t^{\prime} \in \mathbb{R}\right),
$$

where $\sigma$ is the standard symplectic structure on $\mathbb{R}^{2 n}$.

$$
\sigma\left(z, z^{\prime}\right)=\left\langle x, \xi^{\prime}>-<x^{\prime}, \xi>\left(x, x^{\prime} \in \mathbb{R}^{n}, \xi, \xi^{\prime} \in\left(\mathbb{R}^{n}\right)^{*}\right) .\right.
$$


Let "denote again the group generated by, say, left translations and dilations. Its action on $L^{2}(G)$ is given by

$$
\begin{gathered}
f(x) \rightarrow f(h x), \\
f(x) \rightarrow \delta^{\alpha 2} f\left(\gamma_{\delta}(x)\right),
\end{gathered}
$$

and, changing our point fo view, we can take formula (1) in Section 2 as a definition of paracommutator. As a generalization of formula (2) in the same Section, the kernel is then of the form

$$
\int_{G} K\left(y^{-1} x_{1}, y^{-2} x_{2}\right) b(y) d y
$$

where $K$ is homogeneous of degree $-2 Q$ in the sense that

$$
K\left(\gamma_{\delta}\left(x_{1}\right), \gamma_{\delta}\left(x_{2}\right)\right)=\delta^{-2 Q} K\left(x_{1}, x_{2}\right) \quad(\delta>0)
$$

and where we integrate with respect to the Haar measure on $G$. We denote the corresponding operator by $T_{b}=T_{b}(K)$.

In this case we can no longer make use of the Fourier transform or at least it is less suitable here. However, we conjecture that most of the previous theory (see Section 1) carries over to the present situation.

A first concern is then to find the proper analogue of the scale of spaces $=\not_{p}$.

Let $E_{1}, \ldots, E_{r}$ be a basis for the space $V_{1}$ ocurring in the stratification $\left(r=\operatorname{dim} V_{1}\right)$. Then the operator $\gamma=-\left(E_{1}^{2}+\ldots+E_{r}^{2}\right)$ is called the $s u b$-Laplacian of G. Following [F] we can then define (homogeneous) Sobolev spaces $\dot{W}_{p}^{k}(G)$ using complex powers of $\gamma$. By real interpolation and duality one can then define the (homogeneous) Besov spaces $B_{p}^{\bullet}(G)$, at least for $l<p<\infty$. It is now natural to set $-A_{p}=\dot{B}_{p}^{\text {opp.p }}(G)$, which spaces, apparently, come equipped with $t^{\prime}$-invariant norms

$$
\|b o g\|_{\mathcal{A}_{g}}=\|b\|_{\mathcal{A}_{p}} \quad\left(g \in^{\prime} \%\right) .
$$

We now look a little closer at the case $p=2$. The Hilbert-Schmidt norm of the paracommutator $T_{b}$ is then given by

(1) $\left\|T_{b}\right\|_{H S}^{2}=\iint_{G \times G} L\left(y^{-1} z\right) b(y) \overline{b(z)} d y d z$

with formally 


$$
L\left(y^{-1} z\right)=\iint_{G \times G} K\left(y^{-1} x_{k}, y^{-1} x_{2}\right) \overline{K\left(z^{-1} x_{1}, z^{-1} x_{2}\right)} d y d z
$$

this as a generalization of formulae (3)-(4) in Section 2. We must compare

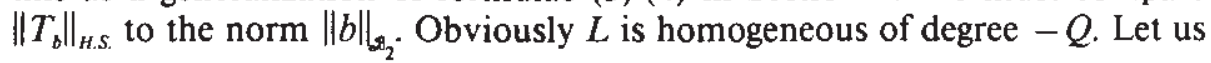
assume that it is $C^{\infty}$ outside the origin. The right hand side of (1) has the form $(L * b, b)$ where $($,$) is the inner product in L^{2}(G)$ and $*$ stands for convolution. In [F], th.2.1 it is shown that the operator $\gamma$ has a unique fundamental solution $\ell$ which is homogeneous of degree $-\mathrm{Q}, \mathcal{\ell} * \gamma=\gamma * \mathcal{f}=\delta$. Therefore we can write the last expression also as

$$
\left(M * \gamma^{Q \prime 2} b, b\right)
$$

where $M$ is homogeneous of degree 0 , this corresponds essentially to a Calderón-Zygmund transformation, bounded in $L^{2}(G)$ in view of [F], prop. 1.9. We conclude that

$$
\left\|T_{b}\right\|_{H . S .} \leqq C\|b\|_{t_{2}}
$$

If we make the assumption that this transformation can be inverted we have also an estimate in the opposite direction. In summary we have thus "proved":

"Theorem". In suitable assumptions on $K$ hold $T_{b}(K) \in S_{2}$ iff $b \in \in_{e} t_{2}$.

To proceed further we need also the minimality of the space $e \mathscr{R}_{1}$ or, what is the same, the maximality of $\mathcal{B}_{\mathbb{\alpha}^{\prime}}$. It is also clear that the assumptions on $\mathrm{K}$ are less explict than in the case of $\mathbb{R}^{n}$, in which case they were formulated in terms of $A$ (see Section 1). We therefore postpone a further treatment to a future occasion.

\section{TRACE IDEAL CLASSES OF MULTILINEAR FORMS}

In the previous sections we have drawn ideas from the theory of function spaces, from abstract interpolation, even from group representations. We conclude with something which is more genuinely functional analysis (more abstract), and thus closer to the heart of the matter of this conference.

The point is that it is often useful not to view paracommutators as operators but rather as bilinear forms. More precisely, with slight change of the meaning of $A$ - we restrict attention to the Abelian case only $\left(G=\mathbb{R}^{n}\right)$ - we may consider the form 


$$
H_{b}(f, g)=\frac{1}{(2 \pi)^{2 n}} \iint_{\mathbb{R}^{n} \times \mathbb{R}^{n}} A\left(\xi_{t}, \xi_{2}\right) \hat{b}\left(\xi_{1}+\xi_{2}\right) \hat{f}\left(\xi_{1}\right) \hat{g}\left(\xi_{2}\right) d \xi_{1} d \xi_{2}
$$

where $f$ and $g$ are elements of $L^{2}\left(\mathbb{R}^{n}\right)$; this form is likewise termed the paracommutator with symbol $b$. Notice that the critical set is now the "anti-diagonal" $\left\{\left(\xi_{1}, \xi_{2}\right): \xi_{1}+\xi_{2}=0\right\} \subset \mathbb{R}^{n} \times \mathbb{R}^{n}$. Because of the essential identity of bilinear forms and operators, one gets and entirely parallel theory. But it is now also natural to look at analogous multilinear objects: trilinear forms of the type

$$
\begin{gathered}
H_{b}(f, g, h)=\frac{1}{(2 \pi)^{3 n}} \iiint_{\mathbb{R}^{n} \times \mathbb{R}^{n} \times \mathbb{R}^{n}} A\left(\xi_{1}, \xi_{2}, \xi_{3}\right) \hat{b}\left(\xi_{1}+\xi_{2}+\xi_{3}\right) \times \\
\quad \times \hat{\jmath}\left(\xi_{1}\right) \hat{g}\left(\xi_{2}\right) \hat{h}\left(\xi_{3}\right) d \xi_{1} d \xi_{2} d \xi_{3}
\end{gathered}
$$

and so forth with any number of arguments. However, as far as we know, it is not clear what is meant by a trace ideal class of multilinear forms. The issue has already been touched upon in [Pee], Chap. 5 and in [JPR], Sec. 5, and we repeat here what was said there, with perhaps a few more details.

There are at least two methods of defining classes of multilinear forms analogous to the usual classes $S_{p}$ in the case of compact linear operators in Hilbert space.

For notational simplicity we consider only the case of trilinear forms.

\section{First method}

Let there be given three Hilbert spaces $H_{1}, H_{2}, H_{3}$. We denote by $S_{\infty}$ the space of all compact bilinear forms on $H_{1} \times H_{2} \times H_{3}$ (with values in C). It is clearly a Banach space (in the supremum norm) and a normed ideal in the sense that if $B=B(x, y, z)$ is in $S_{\infty}$ then so is $B(U x, V y, W z)$ and with the same, or a smaller, norm whenever $U, V, W$ are contractions on $H_{0}, H_{2}, H_{3}$ respectively. We further define $S_{2}$ to be the Hilbert-Schmidt class, that is, $B$ is in $S_{2}$ iff we have $B(x, y, z)=\sum a_{i j k} x_{2} y, z_{k}$ with $\left.\sum a_{i j k}\right|^{2}<\infty$, where $\left\{x_{i}\right\},\{y\},\left\{z_{n}\right\}$ are the coordinates with respect to any orthogonal bases in $H_{1}, H_{2}, H_{3}$. This condition is independent of the basis. Finally, we define $S_{1}$ to be the space of all nuclear forms, i.e. forms $B$ of the type

$$
B(x, y, z)=\Sigma \lambda_{i}\left(x, \xi_{i}\right)\left(y, \eta_{)}\right) \cdot\left(z, \zeta_{i}\right)
$$

where $\left\{\lambda_{i}\right\},\left\{\xi_{i}\right\},\left\{\eta_{i}\right\},\left\{\zeta_{i}\right\}$ are sequences in $\mathrm{C}, H_{1}, H_{2}, H_{3}$ respectively with $\sum\left|\lambda_{1}\right|\left\|\xi_{i}\right\|\left\|\eta_{i}\right\| \| \zeta_{i}||<\infty$. In the same way we define $S_{p}, 0<p<1$, but then we get quasinormed ideals, not normed ideals. 
What about $S_{p}, 1<p<2$ or $2<p<\infty$ ? We suggest defining $S_{p}$ by interpolation, either real or complex, i.e. $S_{p}=\left(S_{1}, S_{\infty}\right)_{\theta, \theta}$ or $S_{p}=\left[S_{1}, S_{\infty}\right]_{\theta}$ where $1 / p=1-\theta$. It is not clear that two definitons coincide, as in the operator case, nor that they agree with the definition of $S_{2}$. A heuristic argument for the support of the latter statement goes as follows: the dual of $S_{\mathrm{co}}$ (in the natural duality provided by the inner product of $S_{2}$ ) is $S_{1}$. Therefore we are in principle in the situation of the "theorem on interpolation between a space and its dual" ([LP]; cf. [BL]). But since $S_{\infty}$ is not reflexive the theorem is unfortunately not directly applicable. Also, it is not clear at all that the entire scale $S_{p} .0<p \leqq \infty$, is closed for interpolation.

\section{Second method}

This is based on the application of Schmidt or approximation numbers. If $B$ is any compact trilinear form we set

$$
S_{n}(B)=\inf _{\text {rankF } \| n}\|B-F\|_{\infty} \quad(n=0,1,2, \ldots),
$$

where the infimum thus runs over all forms $F$ of finite rank $\leqq n$. If $0<p \leqq \infty$ we define $\tilde{S}_{p}$ to be the set of forms $B$ such that the sequence $\left\{s_{n}(B)\right\}$ is in $l_{p}$. It is clear that $\tilde{S}_{p}$ is a quasi-normed ideal. From the general theory of interpolation of normed Abelian groups ([PS]; cf. [BL], Chap. 3 and Chap. 7) it follows at any rate that the scale $\tilde{S}_{p}$ is closed for interpolation:

$$
\tilde{S}_{p}=\left(\tilde{S}_{p_{g}}, \tilde{S}_{p_{1}}\right)_{\theta, \infty} \text { for } 0<\theta<1, \frac{1}{p}=\frac{1-\theta}{p_{0}}+\frac{\theta}{p_{1}} \text {. }
$$

But is it true that $S_{p}=\tilde{S}_{p}$ ? It is not even clear that the spaces $\tilde{S}_{p}$ are Banach spaces for any $p<\infty$.

\section{References}

[A] S. AXLER, The Bergman space, the Bloch space, and commutators of multiplication operators. Duke Math. J. 53 (1986), 315-332

[AFP] J. ARAZY, S. FISHER, J. PEETRE, Hankel operators in Bergman spaces. Am. Math. J. 110 (1988), 989-1053.

[BL] J. BERGH, J. LÖFSTROM Interpolation spaces. An introduction. (Grundlehren 226). Springer, Berlin-Heidelberg-New York, 1976.

[F] G.B. FOLLAND, Subelliptic estimates and function spaces on nilpotent Lie groups. Ark. Mat. 13 (1975), 161-207.

[JP] S. Janson, J. Peetre, Paracommutators - boundedness and Schattenvon Netumann properties. Trans. Am.. Math. Soc. 305 (1988), 467-504. 
[JPR] S. Janson, R. Rochberg, J. Peetre, Hankel forms and the Fock spaces. Rev. Mat. Iberoamer. 3(1987), 61-129.

[LP] J.L. LIONS, J. PeETRE, Sur une classe d'espaces d'interpolation. Inst. Hautes Etudes Sci. Publ. Math. 19(1964), 5-68.

[PS] J. PEETRE, G. SPARR, Interpolation of normed Abelian groups. Ann, Mat. Pure Appl. 92 (1972), 217-262.

[Pee] J. PeEtre, Paracommutators and minimal spaces. In: S.C. Power (ed.), Operators and function theory (Proceedings, Lancaster 1984), pp. 163-224. Reidel, Dordrecht, 1985.

[Pel] V. V. PELLER, Hankel operators of class $S_{p}$ and their applications ( $r a-$ tional approximation, Gaussian processes, the majorant problem for operators). Mat. Sb. 113 (1980), 538-581. [Russian].

[Pen 1] Peng Lizhong, Contributions to certain problems in paracommutators. Dissertation, Stockholm, 1986.

[Pen2] Peng Lizhong, On the compactness of paracommutators. Ark. Mat. 26 (1988), 315-325.

[Pen3] Peng Lizhong, Paracommutators of Schatten-von Neumann class $S_{p}$ $0<\mathrm{p}<1$. Math. Scand. 61 (1987), 68-92.

[QP] QIAN TAO, PENG LizHONG, $A$ kind of multilinear operator and the Schatten-von Neumann classes. Ark. Mat. 27 (1989), 145-154.

[R] R. ROCHBERG, Trace ideal criteria for Hankel operators and commutators. Indiana U. Math. J. 31 (1982), 913-925.

[T1] V. TIMOTIN, $A$ note on $C_{p}$ estimates for certain kernels. Integral Equations Operator Theory $9^{\circ}(1986), 295-304$.

[T2] V. Timotin, $C_{\mathrm{p}}$-estimates for certain kernels: the case $0<\mathrm{p}<1$. J. Funct. Anal. $72^{\circ}$ (1987), 368-380.

[T3] V. Timotin, $C$-estimates for certain kernels on local fields. Studia Math. 88 (1988), 43-50.

Matematiska institutionen

Box 6701

S-11385 Stockholm (SWEDEN)

Departamento de Matemáticas

Universidad Autónoma

28049 Madrid (ESPAÑA) 